\title{
T2DM treatment trial results
}

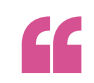

\section{Taken \\ together, these \\ trial results}

are good news

for patients

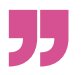

In June 2019, five key papers were published on the safety and efficacy of three drugs for treating type 2 diabetes mellitus (T2DM).

While safety data for injectable semaglutide (a glucagon-like peptide 1 (GLP1) receptor agonist) have been available for some time, information on the new oral form of semaglutide has been lacking. One of the aims of the PIONEER trials was to address this gap. The first study reports results from PIONEER 5, which assessed the safety and efficacy of oral semaglutide in patients with T2DM and moderate renal impairment. (baseline eGFR $30-59 \mathrm{ml} / \mathrm{min} / 1.73 \mathrm{~m}^{2}$ ) The study included 324 patients; 163 received oral semaglutide and 161 received placebo. After 26 weeks of treatment oral semaglutide was shown to reduce $\mathrm{HbA}_{1 \mathrm{c}}$ levels compared with placebo. Although several patients receiving oral semaglutide reported adverse effects (mainly mild-to-moderate nausea), these were consistent with the class effects of GLP1 receptor agonists. The authors therefore conclude that oral semaglutide is safe and effective in patients with moderate renal impairment.

In the PIONEER 6 study, 3,183 patients with T2DM who were at high risk of cardiovascular events were randomly assigned to

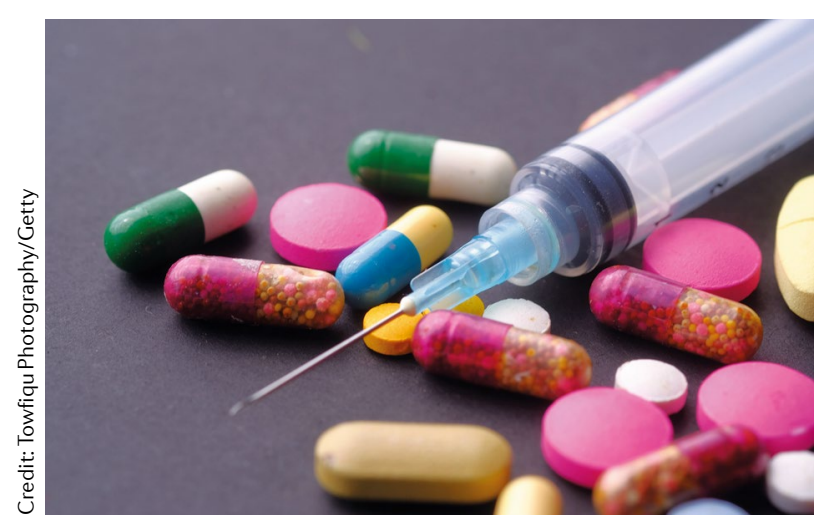

receive either oral semaglutide or placebo. Patients were followed up for a median of 15.9 months so that investigators could assess cardiovascular safety. Over the trial period, 61 of 1,591 patients in the oral semaglutide group experienced major cardiovascular events, compared with 76 of 1,592 patients in the placebo group. Thus, oral semaglutide is not inferior to placebo in terms of cardiovascular risk.

The PIONEER 7 study compared oral semaglutide with flexible dose adjustment to sitagliptin (a dipeptidyl peptidase 4 inhibitor and established T2DM therapeutic). The trial included 504 patients with T2DM who were randomly assigned to receive one of the regimens and were followed up for 52 weeks. Over the study period, patients receiving oral semaglutide achieved better glycaemic control and lost more weight than patients on sitagliptin. In addition, the safety profile of oral semaglutide was similar to that of injectable GLP1 receptor agonists.

The REWIND trial has now reported on the cardiovascular efficacy of another GLP1 receptor agonist - dulaglutide. This international study included 9,901 patients who had T2DM with (31.5\%) or without $(68.5 \%)$ evidence of previous cardiovascular disease (CVD). The patients were randomly assigned to receive either dulaglutide or placebo and were followed up for a median of 5.4 years. Over this period, major cardiovascular events occurred in $12 \%$ of patients in the dulaglutide group and $13.4 \%$ of patients in the placebo group, with similar effects in those with and without previous CVD. The authors therefore suggest that dulaglutide is a treatment option for middle aged and older adults with T2DM who are at risk of cardiovascular events and are similar to those seen in routine clinical practice.

The fifth trial (DECLARETIMI 58) assessed the effect of dapagliflozin (a sodium-glucose cotransporter 2 inhibitor) on kidney disease in patients with T2DM who either did or did not have atherosclerotic CVD. The study included 17,160 patients who were randomly assigned to receive either dapagliflozin or placebo and were followed up for a median of 4.2 years. It was found that decline in eGFR was slower in the dapagliflozin group than in the placebo group, and risk of end-stage renal disease or renal-associated death was also lower in the dapagliflozin group. The authors conclude that dapagliflozin can prevent the development of kidney disease and reduce its progression in those who already have kidney disease, compared with placebo.

Taken together, these trial results are good news for patients, who will hopefully soon have more options for managing their T2DM.

Claire Greenhill

ORIGINAL ARTICLE Mosenzon, O. et al. Efficacy and safety of oral semaglutide in patients with type 2 diabetes and moderate renal impairment (PIONEER 5): a placebo-controlled, randomised, phase 3a trial. Lancet Diabetes Endocrinol. https:// doi.org/10.1016/S2213-8587(19)30192-5 (2019) | Husain, M. et al. Oral semaglutide and cardiovascular outcomes in patients with type 2 diabetes. N. Engl.J. Med. https://doi.org/10.1056/ NEJMoa1901118 (2019)|Pieber, T. R. et al. Efficacy and safety of oral semaglutide with flexible dose adjustment versus sitagliptin in type 2 diabetes (PIONEER 7): a multicentre, open-label, randomised, phase 3a trial. Lancet Diabetes Endocrinol. https:// doi.org/10.1016/S2213-8587(19)30194-9 (2019)| Mosenzon, O. et al. Effects of dapagliflozin on development and progression of kidney disease in patients with type 2 diabetes: an analysis from the DECLARE-TIMI 58 randomised trial. Lancet Diabetes Endocrinol. https://doi.org/10.1016/ S2213-8587(19)30180-9 (2019)|Gerstein, H. C. et al. Dulaglutide and cardiovascular outcomes in type 2 diabetes (REWIND): a double-blind, randomised placebo-controlled trial. Lancet https://doi.org/ 10.1016/S0140-6736(19)31149-3 (2019) 